\title{
On the Zeroth-Order Hamiltonian for CASPT2 Calculations of Spin Crossover Compounds
}

Sergi Vela, ${ }^{*[a, b]}$ Maria Fumanal, ${ }^{[b]}$ Jordi Ribas-Ariño, ${ }^{[b]}$ and Vincent Robert ${ }^{[a]}$

[a] S. Vela, V. Robert Laboratoire de Chimie Quantique, UMR 7177, Université de Strasbourg, 4 rue Blaise Pascal, F-67000 Strasbourg, France

[b] S. Vela, M. Fumanal, J. Ribas-Ariño, Departament de Química Física and IQTCUB, Universitat de Barcelona, Av. Diagonal 645, 08028, Barcelona, Spain E-mail: sergi.vela@gmail.com 


\begin{abstract}
Complete active space self-consistent field theory (CASSCF) calculations and subsequent second-order perturbation theory treatment (CASPT2) are discussed in the evaluation of the spin-states energy difference $\left(\Delta H_{\mathrm{elec}}\right)$ of a series of seven spin crossover (SCO) compounds. The reference values have been extracted from a combination of experimental measurements and DFT $+U$ calculations, as discussed in a recent article (Vela et al., Phys Chem Chem Phys 2015, 17, 16306). It is definitely proven that the critical IPEA parameter used in CASPT2 calculations of

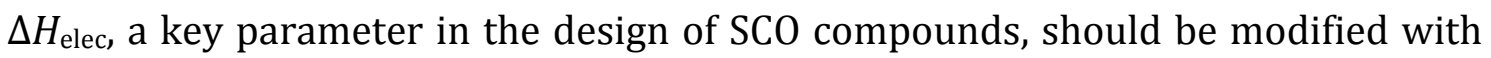
respect to its default value of 0.25 a.u. and increased up to 0.50 a.u. The satisfactory agreement observed previously in the literature might result from an error cancellation originated in the default IPEA, which overestimates the stability of the HS state, and the erroneous atomic orbital basis set contraction of carbon atoms, which stabilizes the LS states.
\end{abstract}




\section{Introduction}

Spin crossover compounds (SCO) are fascinating materials from the point of view of quantum chemistry.1-3 Their ground and first-excited spin states may be interchanged upon application of a variety of triggering effects such as light, pressure or temperature, being the latter the most common. ${ }^{4,5}$ For instance, when those materials are based on an $\mathrm{Fe}^{\mathrm{II}}$ ion, the low-lying spin states correspond to a singlet (Low-Spin, LS, $S=0$ ) and a quintuplet (High Spin, HS, $S=2$ ). ${ }^{6}$ Typically, the LS state is favored by enthalpy $(\Delta H)$, whereas the HS state is stabilized by the larger vibrational and electronic entropy $(\Delta S)$ that results from the population of antibonding Metal-Ligand bonds and the electron unpairing, respectively. Due to the nature of the two spin states involved and the small energy difference between them, it becomes a challenging task to quantify their relative stability, or even elucidating which is the ground state. To this purpose, CASPT2 (second-order perturbation theory on top of a Complete Active Space self-consistent field calculation) has been the workhorse of the chemistry community to accurately calculate $\Delta H_{\text {elec }}$ in isolated SCO molecules, and has been successful in the quantification of adiabatic ${ }^{7,8}$ and vertical energy differences. ${ }^{9}$ However, in the design of CASPT2 methodology, an IPEA parameter had to be incorporated to correct the energy of the electron excitations from (and to) the active space orbitals. Its default value of 0.25 atomic units (a.u.), proposed by Ghigo et al. in $2004,{ }^{10}$ was obtained after analyzing the performance of the CASPT2 method to reproduce the dissociation energies of several binuclear species and the excitation energies of $\mathrm{N}_{2}$ and benzene and, thus, seems far from the spin state problem tackled in the quest for $\Delta H_{\text {elec }}$. This issue has carried some controversy: recent studies by Kepenekian et al.11 and Lawson Daku et al. ${ }^{12}$ have advocated for the use of larger IPEA shifts, whereas Rudavsky et al. ${ }^{7}$ found that a better agreement is achieved with the default value. Whatever the case, it does not seem a good idea to develop a rather sophisticated methodology such as CASPT2, and leave its energetic accuracy depend on a parameter that has been only benchmarked for one type of problematic. Of course this problem is only relevant when very tiny energy differences are involved (few tens of $\mathrm{kJ} \mathrm{mol}^{-1}$ ), but this is precisely the case of adiabatic energy gaps in SCO compounds. 
In the present article we will provide definitive evidence that the adequate IPEA value to study this class of compounds must be increased up to about 0.5 a.u., twice the value proposed originally. The main advance of this work with respect to Refs. [11] and [12] is that very recently, in a article devoted to the application of the DFT $+U$ approach to study SCO compounds, ${ }^{13}$ we obtained accurate estimates of the thermodynamic magnitudes of interest, including $\Delta H_{\mathrm{elec}}$, thereby obtaining sound reference values and allowing for an accurate benchmark of the IPEA parameter. Furthermore, our new calculations imply other advantages. (i) They have been performed in other SCO molecules never analyzed before, thus extending the number of benchmarked molecules. (ii) We have used molecular geometries optimized in the solid-state, thus eliminating any possible error associated to gas-phase geometries. (iii) Finally, and more important, they take into account the recently-discovered error ${ }^{14}$ in the relativistic ANO-RCC basis set for $\mathrm{C}$ atoms used in Molcas. ${ }^{15}$ We will comment on the importance of this technical error on the SCO studies performed so far with this computational package, and demonstrate that, fortuitously, it actually contributed to diminish the error associated with the standard IPEA value in previous inspections.

This manuscript is organized as follows. In the methods section, we present the strategy that we followed in ref. 13 to extract the thermodynamic magnitudes from experimental data, the computational details, and an introduction to the IPEA parameter. We must note here that the seven compounds used as a dataset in this paper are the same that we used in this recent manuscript, with chemical formulae: Fe(phen $)_{2}(\mathrm{NCS})_{2}(\mathbf{1}),{ }^{15}\left[\mathrm{Fe}(\mathrm{abpt})_{2}(\mathrm{NCS})_{2}\right](\mathbf{2}),{ }^{16}\left[\mathrm{Fe}(\mathrm{abpt})_{2}(\mathrm{NCSe})_{2}\right](\mathbf{3}),{ }^{16}$ Fe(bapbpy)(NCS) $)_{2}(4),{ }^{17} \mathrm{Fe}\left(\mathrm{HB}(\mathrm{pz})_{3}\right)_{2}(5),{ }^{18} \mathrm{Fe}\left[\mathrm{H} 2 \mathrm{~B}(\mathrm{pz})_{2}\right]_{2}$ (bipy) (6) ${ }^{19}$ and $[\mathrm{Fe}(1-$ bpp)][BF $]_{2}(7) .{ }^{20}$ Those compounds have a common structural characteristic, they display a $\mathrm{FeN}_{6}$ core, probably the largest family of inorganic SCO materials (see Figure 1). ${ }^{6}$ Then, in the results section, we will present and discuss the results; in particular, we will definitely prove that an IPEA value of ca. 0.5 a.u. is more adequate to represent the spin state energetics of this class of materials, with some exceptions that we will comment and, finally, we will summarize the conclusions that can be extracted from this piece of work. 


\section{Methods}

A thermally-driven spin transition occurs when the higher enthalpy of the LS state is overcome by the favorable entropy contribution of the HS state at $T_{1 / 2}$. At this temperature, $\Delta G$ is equal to zero and the transition temperature $T_{1 / 2}$ can be obtained from:

$$
T_{1 / 2}=\frac{\Delta H_{t o t}\left(T_{1 / 2}\right)}{\Delta S_{t o t}\left(T_{1 / 2}\right)}
$$

where $\Delta X=X(H S)-X(L S)$. As defined in statistical thermodynamics, both enthalpy and entropy can be decomposed in multiple terms accounting for electronic, rotational, vibrational and translational differences between the two spin states involved in the transition. Usually, the contribution of the rotational and translational terms is neglected, as they are not expected to contribute significantly, and only the electronic and vibrational terms are taken into account (eq. 2 and 3).

$$
\begin{gathered}
\Delta H_{\text {tot }}(T)=\Delta H_{\text {elec }}+\Delta H_{\text {vib }}(T) \\
\Delta S_{\text {tot }}(T)=\Delta S_{\text {elec }}+\Delta S_{\text {vib }}(T)
\end{gathered}
$$

We must note here that the value that is usually computed as the energy difference between spin states corresponds to $\Delta H_{\text {elec }}$, and once the vibrational correction $\Delta H_{v i b}$ is included, the resulting magnitude can be compared to the experimental enthalpy estimate $\Delta H_{\text {tot }}$ obtained usually from DSC measurements. In recent work, we have described a strategy to extract the $\Delta H_{\text {elec }}$ directly from experimental data and after the evaluation of $\Delta H v i b, \Delta S v i b$ and $\Delta S e l e c .{ }^{13}$ We did so for compounds 1-7 and used the obtained $\Delta H_{\text {elec }}$ values as a reference for the parametrization of the Hubbard-like U-term that best describes the energetics of those compounds within the DFT+U framework. As a result of this study, we were able to obtain accurate $\Delta H_{\text {elec }}$ values of the studied SCO molecules both in the crystalline phase and in the gas phase (isolated), using the best parametrization of $U$ to conduct this 
study. The original purpose of evaluating $\Delta H_{\text {elec }}$ in isolated molecules was to quantify the effect of the intermolecular interactions. However, the obtained $\Delta H_{\text {elec }}$

values for the isolated molecules, hereafter referred to as $\Delta H_{\mathrm{elec}}^{\text {ref }}$, can be also used to analyze the performance of different computational methods working in gas phase. Within this context, in the present paper we have fine-tuned the IPEA parameter used in the CASPT2 approach to reproduce those $\Delta H_{\mathrm{elec}}^{\text {ref }}$ values. The reader is referred to ref. 13 for further details on how the $\Delta H_{\text {elec }}^{\text {ref }}$ values were obtained.

\section{Computational details}

\section{Molecular geometries}

The molecular geometries upon which the CASPT2 treatment was subsequently applied were obtained by performing a variable-cell geometry optimization of the crystalline phase of the SCO compounds at the corresponding spin state. Those calculations were performed using the Quantum Espresso package $(\mathrm{QE}),{ }^{21}$ the PBE functional within the spin unrestricted formalism, the D2 correction of Grimme, Vanderbilt pseudopotentials, a $\Gamma$-point sampling of the Brillouin zone. For the variable-cell geometry calculations we used a Hubbard-like $U$ term of $2.5 \mathrm{eV}$, applied on the $d$ orbitals of Fe, within the DFT+U methodology. This value was selected prior to its benchmark based on adiabatic energy differences $(U=2.65$ $\mathrm{eV}$ ), but we could confirm that the use of $U=2.5 \mathrm{eV}$ or $U=2.65 \mathrm{eV}$ yielded the same crystalline structures.

\section{CAS $(10,12)$ and CASPT2 calculations}

Using those optimized geometries, a complete active space self-consistent field (CASSCF, also referred to as CAS) calculations have been converged for all HS and LS states. All active spaces include the $3 \mathrm{~d}$ and $4 \mathrm{~d}$ orbitals of iron, together with the $\mathrm{N}(\mathrm{p})-\mathrm{Fe}(\mathrm{d}) \sigma$-orbitals, yielding a total of 10 electrons in 12 orbitals, namely CAS(10,12). This active space has been proposed in the literature $9,22,23$ to be adequate to describe such materials, since it includes the non- dynamical (or 
static) correlation of the Fe atom, as well as the most important contributions to dynamical correlation. Then, using the $\operatorname{CAS}(10,12)$ wavefunction, all the remaining dynamic correlation has been included with a posterior CASPT2 treatment on the whole set of orbitals, excluding the deep-core orbitals (1s of $C, N$ and B, 1s, 2s, 2p, $3 s$ and $3 p$ of Se, and $1 s, 2 s$ and $2 p$ of Fe and S).

In CASPT2, the zeroth-order Hamiltonian is built using one-electron Fock-type operators $^{24}$ and, according to this formulation, the diagonal terms of the Fock matrix can be written as ${ }^{10}$ :

$$
F_{p p}=-\frac{1}{2}\left(D_{p p}(I P)_{p}+\left(2-D_{p p}\right)(E A)_{p}\right)
$$

where $D_{p p}$ is the occupation of orbital $p$, and IP and EA are its ionization potential and electron affinity, respectively. For empty and fully-occupied orbitals, $F_{p p}$ becomes, respectively, EA and IP, whereas for partially-occupied orbitals, one obtains:

$$
F_{p p}=-\frac{1}{2}\left((I P)_{p}+(E A)_{p}\right)
$$

However, it was seen in the early days of the CASPT2 method that this formulation overestimated the stability of open-shell systems and that, instead, it was preferable to obtain $F_{p p}=-I P$ for excitations from a partially-occupied orbital and $F_{p p}=-E A$ for excitations into a partially-occupied orbital. In order to remove this systematic error, a modification of the zeroth-order Hamiltonian was proposed and the following shifts were added to the diagonal terms of the Fock matrix (ie. $F_{p p}$, see eq. 4$):$

$$
\begin{gathered}
\sigma_{p}^{(E A)}=\frac{1}{2} D_{p p}\left((I P)_{p}-(E A)_{p}\right)=\frac{1}{2} D_{p p} \varepsilon \\
\sigma_{p}^{(I P)}=-\frac{1}{2}\left(2-D_{p p}\right)\left((I P)_{p}-(E A)_{p}\right)=-\frac{1}{2}\left(2-D_{p p}\right) \varepsilon
\end{gathered}
$$


The first one (eq.6) is applied when exciting into an active orbital and the second (eq.7), when exciting out of this active orbital, therefore resulting in the desired $E A$ and - IP energies, respectively. Given that the definition of $(I P)_{p}$ and $(E A)_{p}$ is difficult, their energy difference is replaced by a single averaged parameter, namely the IPEA shift ( $\varepsilon$ in eq. 6 and 7 ) and the scope of this work is, precisely, to determine the adequate value of this parameter to obtain the correct adiabatic energy differences between the LS and HS state of $\mathrm{Fe}^{\mathrm{II}}-\mathrm{N}_{6}$ based SCO compounds.

All CASSCF-CASPT2 calculations have been carried out with the Molcas 8.0 package. We have used ANO-RCC basis sets, designed to include the scalar relativistic effects with the following contractions: [3s2p1d] for $\mathrm{C}^{25}$ [7s6p5d3f2g] for $\mathrm{Fe}^{25}[2 \mathrm{~s}]$ for $\mathrm{H}^{26}[4 \mathrm{~s} 3 \mathrm{p} 1 \mathrm{~d}]$ for $\mathrm{S}^{27}[5 \mathrm{~s} 4 \mathrm{p} 2 \mathrm{~d} 1 \mathrm{f}]$ for Se, ${ }^{27}[3 \mathrm{~s} 2 \mathrm{p} 1 \mathrm{~d}]$ for $\mathrm{B}^{27}$ and, finally, we have divided the $\mathrm{N}$ atoms in two groups. (i) Those coordinated to Fe, which have been described using the [4s3p2d1f] contraction, and (ii) the remaining atoms, for which we have used the [4s3p1d] contraction. ${ }^{27}$ It must be noted that this level of contraction in the basis set has been found to be a good compromise between accuracy and computational cost. ${ }^{22}$ The Cholesky decomposition has been employed to treat the two-electron integrals in all the calculations. Notice that the use of the erroneous (e) or the corrected (c) basis set for the $\mathrm{C}$ atoms does not imply a change in its contraction. In this sense, it is known that larger contractions would lead to smaller differences between $\triangle H_{\text {elec }}^{I P E A, e}$ and $\Delta H_{\mathrm{elec}}^{I P E A, c}$. 


\section{Results}

Most CASPT2 calculations of SCO systems in the quantum chemistry community have been traditionally performed using the Molcas package. ${ }^{14}$ Very recently, an error has been detected on the definition of the relativistic ANO-RCC basis set for carbon atoms, which has been present in versions $6.4-8.0$ of this program, that span 2006-2014. Although it is now corrected, this error has been present in the wide majority of the CASPT2 calculations performed for SCO systems. However, the results obtained using this methodology have been quite successful and, thus, one can intuitively think that its effect on the energetics of different spin-states and, consequently, on $\Delta H_{e l e c}$, must be small. Moreover, one would expect that the $\mathrm{C}$ atoms do not strongly affect the energy of the spin states, since there is no direct bonding or coordination between Fe and C atoms in FeN6-based SCO molecules. In this section we will first demonstrate that the error in the ANO-RCC basis set of C is, on the contrary, quite important, and that is has been somehow hidden by the use of the default IPEA value. The combination of the erroneous basis-set (e) and the default IPEA (0.25 a.u.) ( $\left.\Delta H_{\text {elec }}^{0.25, e}\right)$ has generally worked pretty well due to an error-cancellation that will be carefully analyzed. Therein we will demonstrate that the combination of the corrected basis set (c) and the default IPEA=0.25 a.u. $\left(\Delta H_{\text {elec }}^{0.25, c}\right)$ leads to an increased disagreement of the obtained electronic enthalpy and the reference values ( $\Delta H_{\text {elec }}^{\text {ref }}$, see section 2). Finally, in subsection 3.2 we will benchmark the adequate IPEA that must be combined with the corrected ANO-RCC basis set to achieve quantitative agreement for each of the seven studied compounds. In this sense, all along the results sections we have evaluated the quality of a given approach in terms of the absolute error, and if it successfully predicts the ground spin state of the material ( $\mathrm{P}$ or 0 ). We would like to stress that the values calculated in this manuscript do not incorporate the contribution of the vibrational enthalpy $H_{v i b}$, nor that of the intermolecular interactions, that we quantified in ca. $-5.7 \mathrm{~kJ} / \mathrm{mol}$ and ca. $\pm 5 \mathrm{kcal} / \mathrm{mol}$, respectively, in previous work. ${ }^{13}$

\section{Default IPEA analysis}


A first set of calculations has been performed using the default IPEA parameter (0.25 a.u.) and the erroneous ANO-RCC basis set ( $\Delta H_{\text {elec }}^{0.25, e}$, see Table 1 and Figure 2). The results are moderately-good, with four ground spin states properly characterized (compounds with $\boldsymbol{V}$ in Table 1) and a mean absolute error (MAE) of $13.6 \mathrm{~kJ} / \mathrm{mol}(\sim 0.14 \mathrm{eV})$. However, our analysis clearly reflects that, in general, the HS state is over-stabilized using this parameterization. Those incorporate two errors that will be analyzed in this paper: (i) the parametrization of the IPEA shift, and (ii) the basis set. In order to clarify the contribution of both errors, we have performed the same type of calculations using the corrected ANO-RCC basis set for $\mathrm{C}$, recently included in a newer version of Molcas $\left(\Delta H_{\text {elec }}^{0.25, c}\right.$, see Table 1 and Figure 2 ). The agreement of this set of results is clearly worse than the one shown in Table 1 for the $\Delta H_{\text {elec }}^{0.25, e}$ values. More precisely, the MAE is now $20.6 \mathrm{~kJ} / \mathrm{mol}(\sim 0.21$ $\mathrm{eV}$ ) and the the correct ground state is only predicted for two compounds (5 and 7). The comparison of the results shown so far indicates that the error associated to the basis set is ca. $-7.1 \mathrm{~kJ} / \mathrm{mol}$ (ie. the MAE associated to $\Delta H_{\text {elec }}^{0.25, e}-\Delta H_{\text {elec }}^{0.25, c}$ ), with an even larger over-stabilization of the HS state. In any case, once the error in the basis set is corrected, the MAE obtained for those seven compounds must be only ascribed to the quality of the IPEA parametrization. Therefore, one can immediately see that the default IPEA of 0.25 a.u. is not adequate to study the spinstate energetics of SCO compounds. Indeed, for some compounds the associated error is more than three times larger than the reference electronic enthalpy $\left(\Delta H_{\mathrm{elec}}^{\text {ref }}\right)$ itself.

\section{Extraction of benchmarked IPEA parameter and comparison.}

At this point, once we have demonstrated the important errors associated to the default IPEA parameter, we now benchmarked its value to reproduce $\Delta H_{\mathrm{elec}}^{\text {ref }}$. New CASPT2 calculations have been performed to obtain the evolution of $\Delta H_{\text {elec }}$ as a function of the IPEA parameter, within the range of 0.0 to 0.75 a.u. (see Table 2). Then, a second order polynomial equation has been used to interpolate the exact IPEA value that reproduces $\Delta H_{\text {elec }}$. The resulting benchmarked IPEA parameters lie between 0.35 a.u. and 0.66 a.u., with an average value of 0.53 a.u. (see Table 2). Interestingly, one may notice that the seven values seem to be distributed into two 
subsets; for compounds 1-4 and 6, its value is ca. 0.6 a.u., whereas for compounds 5 and 7, it is considerably lower (ca. 0.35 a.u.). This observation will be analyzed in more detail in the following paragraphs but, in any case, it becomes clear that it is necessary to use an IPEA value larger than the default ( 0.25 a.u.), and closer to 0.5 a.u. The use of this average value to describe the spin gap of the seven SCO compounds leads to a MAE of $6.6 \mathrm{~kJ} / \mathrm{mol}$ (see Table 3 and Figure 2), significantly better than the one obtained in the previous section using a 0.25 a.u. value $(20.6$ $\mathrm{kJ} \cdot \mathrm{mol}^{-1}$, see Table 1). Furthermore, and more important, the ground spin state is correctly described for all compounds except for $\mathbf{2}$.

In order to understand why we have obtained two subsets of benchmarked values, we have analyzed the energy contribution of all types of excitations as a function of the IPEA shift for all compounds and spin states. Interestingly, the energy contribution of the different types of excitations is always modified by roughly the same amount, irrespectively of the compound studied and within a spin state, which indicates that the IPEA shift is actually having the same effect on all $\Delta H_{\text {elec }}$ values not only qualitatively but also quantitatively. For instance, the energy difference between $\Delta H_{\text {elec }}^{0.0, c}$ and $\Delta H_{\text {elec }}^{0.25, c}$ is ca. $23 \mathrm{~kJ} \cdot \mathrm{mol}^{-1}$ for all compounds and, similarly, the differences between $\Delta H_{\text {elec }}^{0.0, c}$ and $\Delta H_{\text {elec }}^{0.75, c}$ are, respectively, ca. 41 $\mathrm{kJ} \cdot \mathrm{mol}^{-1}$, and ca. $58 \mathrm{~kJ} \cdot \mathrm{mol}^{-1}$ (values can be extracted from Table 2). Therefore, one cannot ascribe the appearance of the two subgroups of values to a different behavior of the IPEA shift on the excitation energies. This leads to the conclusion that the origin for such observation is either found in wrong reference values for compounds $\mathbf{5}$ and $\mathbf{7}$, or in the intrinsic behavior of the CASPT2 method.

Regarding the first possibility, one might have doubts on the quality of the reference value that we have taken for compound $\mathbf{5}$, since the $U$ value benchmarked for this compound in ref. 13 (2.97 eV) held also the largest deviation from the mean value arising from the seven evaluated compounds (2.65 eV). Considering that all $\mathrm{FeN}_{6}$-based compounds should present similar $\mathrm{U}$ and IPEA parameters, the fact that both benchmarked values are more deviated from the average in the case of compound $\mathbf{5}$, might be taken as an indication that the reference value adopted to that purpose might be erroneous. Indeed, if $\Delta H_{\mathrm{elec}}^{r e f}$ for $\mathbf{5}$ 
was ca. $35 \mathrm{~kJ} / \mathrm{mol}$ instead of $22.9 \mathrm{~kJ} / \mathrm{mol}$, the resulting $U$ and IPEA values would be both in line with those benchmarked for the remaining compounds. However, the

hypothesis of a bad $\Delta H_{\mathrm{elec}}^{\text {ref }}$ value does not seem to hold for compound $\mathbf{7}$ for two reasons: first, because its benchmarked $U$ value was, in contrast to that of compound 5, similar to the average value (2.60 vs. $2.65 \mathrm{eV}$ ) and, second, because the reference value was successfully used in a previous study ${ }^{28}$ to explain the characteristics of its crystal packing, which would have not been possible if such a large error were to be present. In turn, we have seen that the disagreement of the CASPT2 energies obtained without any IPEA shift $\left(\Delta H_{\text {elec }}^{0.0, c}\right)$, with respect to $\Delta H_{\text {elec }}^{\text {ref }}$ is already smaller for compounds 5 and 7 (ca. $30 \mathrm{~kJ} / \mathrm{mol}$, subtracting $\Delta H_{\text {elec }}^{0.0, c}-\Delta H_{\text {elec }}^{r e f}$ ) than for the remaining ones ( $c a .50 \mathrm{~kJ} / \mathrm{mol}$ ). Therefore, the fact that compounds 5 and 7 require a smaller IPEA value is just because the unmodified zeroth-order Hamiltonian (IPEA=0.0 a.u.) is already showing a better performance for them. In conclusion, we cannot provide any clear explanation for the presence of this two subgroups of benchmarked IPEA values. However, it is worth commenting that such scenario was also found in ref. 11, in which one of the six compounds analyzed therein also displayed a notably different behavior, with an adequate IPEA value that was estimated to be ca. 0.2 a.u.

\section{Conclusions}

The objective of the present paper was to provide a sound benchmark of the adequate IPEA parameter to be used when describing the spin state energetics of FeN6-based SCO compounds. To do so, we have first analyzed the performance of CASPT2 using the default value of 0.25 a.u., and seen that the mean absolute error (MAE) associated to this scheme is $20.6 \mathrm{~kJ} / \mathrm{mol}$. This value was accidentally improved to $13.6 \mathrm{~kJ} / \mathrm{mol}$ due to an error in the definition of the ANO-RCC basis set for $\mathrm{C}$ atoms present in the previous versions of the Molcas package. In any case, the default parameterization implied a relative overestabilization of the HS state, which may reach $300 \%$ of the reference value itself and, as a consequence, the spin state ordering was correctly described only for 2 compounds. After, we have 
identified the IPEA parametrization that reproduces the reference adiabatic energy differences $\left(\Delta H_{\mathrm{elec}}^{\text {ref }}\right)$ of the seven studied compounds, and seen that a value of ca. 0.5 a.u. reduces the error of the CASPT2 method up to a MAE of $6.6 \mathrm{~kJ} / \mathrm{mol}$. More importantly, the ground state of all but one of the studied compounds are correctly described under this parametrization. Notably interesting has been the appearance of two subgroups of benchmarked IPEA parameters centered in notably different values (ca. 0.6 and 0.37 a.u.) that we have traced down to the accuracy of the CASPT2 methodology itself.

Overall, this study demonstrates the moderately-good accuracy of the CASSCFCASPT2 method in its default implementation, since a MAE of ca. $20 \mathrm{~kJ} / \mathrm{mol}$ is far better than that of the most-standard DFT functionals, but also advocates for the adoption of larger IPEA values in order to improve even more the quality of the results and to correctly predict the ground state of this class of compounds. As commented in the introduction, this paper is benefited from the recent publication of sound reference values for the adiabatic energy differences between the HS and LS states of seven SCO compounds $\left(\Delta H_{\mathrm{elec}}^{\text {ref }}\right)$, which is the most important difference with respect to other similar papers ${ }^{11,12}$ that also advocated for the adoption of IPEA=0.5 a.u. Finally, we encourage the implementation of the NEVPT2 scheme, ${ }^{29-}$ 31 a most-promising and parameter-free alternative to CASPT2, in a robust computational package capable to handle the size and the number of orbitals associated to the typical SCO architectures. 
(1)

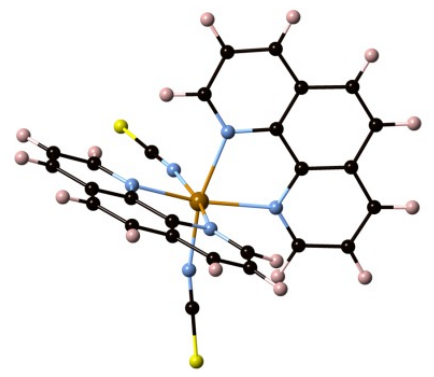

(4)

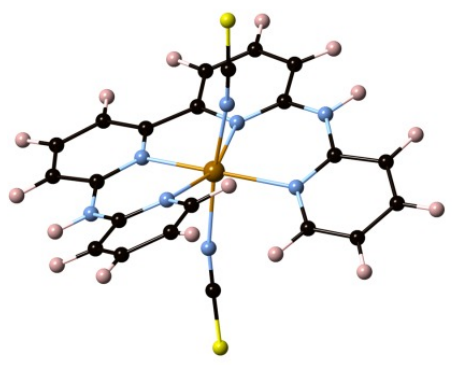

(2)

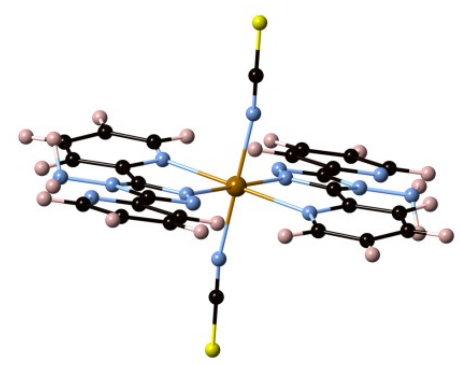

(5)

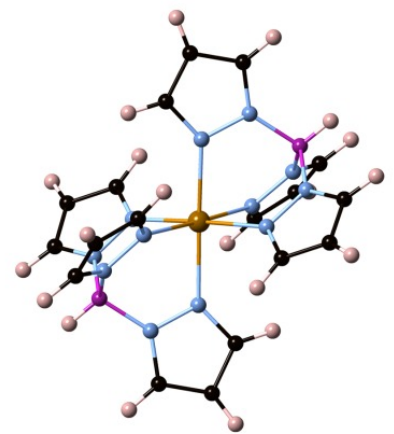

(7)

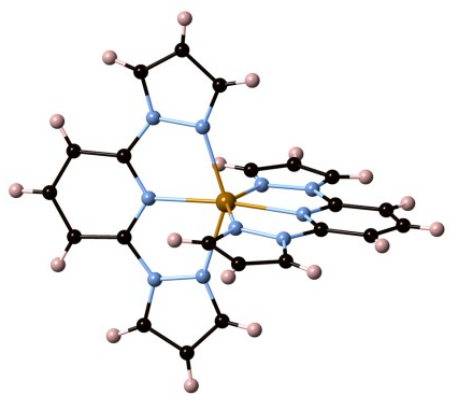

(3)

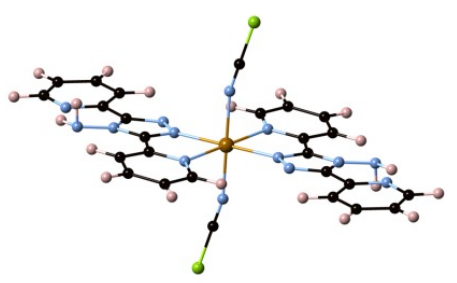

(6)

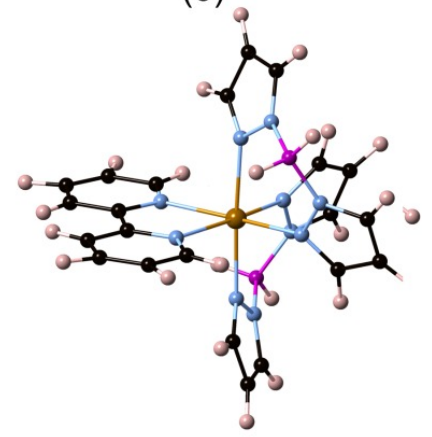

Figure 1. High-spin structures of the seven compounds studied herein, namely: $\mathrm{Fe}(\text { phen })_{2}(\mathrm{NCS})_{2}(\mathbf{1}),\left[\mathrm{Fe}(\mathrm{abpt})_{2}(\mathrm{NCS})_{2}\right](\mathbf{2}),\left[\mathrm{Fe}(\mathrm{abpt})_{2}(\mathrm{NCSe})_{2}\right](\mathbf{3}), \mathrm{Fe}(\mathrm{bapbpy})(\mathrm{NCS})_{2}(\mathbf{4})$, $\mathrm{Fe}\left(\mathrm{HB}(\mathrm{pz})_{3}\right)_{2}(\mathbf{5}), \mathrm{Fe}\left[\mathrm{H}_{2} \mathrm{~B}(\mathrm{pz})_{2}\right]_{2}$ (bipy) (6) and [Fe $\left.{ }^{\mathrm{II}}(1-\mathrm{bpp})\right]\left[\mathrm{BF}_{4}\right]_{2}(\mathbf{7})$. 

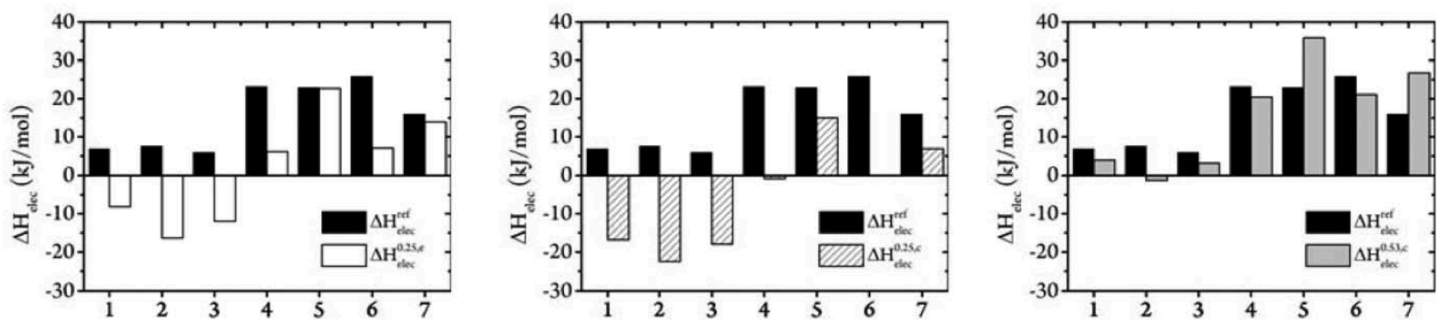

Figure 2. Comparison between the reference adiabatic energy differences ( $\Delta H_{\text {elec }}^{\text {ref }}$, black) and (left) the values obtained with the default IPEA of 0.25 and the erroneous basis-set ( $\Delta H_{\text {elec }}^{0.25, e}$, white), (middle) IPEA 0.25 a.u. and the corrected basis-set ( $\Delta H_{\text {elec }}^{0.25, c}$, stripes), and (right) IPEA 0.53 a.u. and the corrected basis set ( $\Delta H_{\text {elec }}^{0.53, c}$, light gray). 
Table 1: Comparison between reference adiabatic energy values $\left(\Delta H_{\mathrm{elec}}^{\text {ref }}\right)$ elec and those obtained with the default IPEA parameter $(0.25$ a.u.) and with the erroneous (e) and corrected (c) basis set.

\begin{tabular}{|cc|cc|cc|} 
& $\boldsymbol{\Delta H}_{\text {elec }}^{\text {ref }}$ & $\boldsymbol{\Delta H}_{\text {elec }}^{\mathbf{0 . 2 5}, \boldsymbol{e}}$ & Error & $\mathbf{\Delta H}_{\text {elec }}^{\mathbf{0 . 2 5}, \boldsymbol{c}}$ & Error \\
\hline $\mathbf{1}$ & 6.9 & $-8.2(\mathbf{x})$ & -15.1 & $-16.8(x)$ & -23.6 \\
$\mathbf{2}$ & 7.6 & $-16.4(x)$ & -24.0 & $-22.5(x)$ & -30.1 \\
$\mathbf{3}$ & 5.9 & $-12.0(x)$ & -17.9 & $-18.0(x)$ & -23.9 \\
$\mathbf{4}$ & 23.2 & $6.1(\checkmark)$ & -17.1 & $-0.8(x)$ & -24.0 \\
$\mathbf{5}$ & 22.9 & $22.7(\checkmark)$ & -0.2 & $15.0(\checkmark)$ & -7.9 \\
$\mathbf{6}$ & 25.9 & $7.1(\checkmark)$ & -18.9 & $-0.1(x)$ & -26.0 \\
$\mathbf{7}$ & 15.9 & $13.9(\checkmark)$ & -1.9 & $6.9(\checkmark)$ & -9.0 \\
\hline
\end{tabular}

Table 2: Comparison between reference adiabatic energy values $\left(\Delta H_{\mathrm{elec}}^{\text {ref }}\right)$ and those obtained with different IPEA values (from 0.0 to 0.75 a.u.) and the corrected (c) basis set. Also given the IPEA value (in a.u.) that reproduces $\Delta H_{\mathrm{elec}}^{r e f}$. All energy values are given in $\mathrm{kJ} \cdot \mathrm{mol}^{-1}$.

\begin{tabular}{c|cccc|c|c} 
& $\boldsymbol{\Delta H}_{\text {elec }}^{\mathbf{0 . 0}, \boldsymbol{c}}$ & $\boldsymbol{\Delta H}_{\text {elec }}^{\mathbf{0 . 2 5 ,}}$ & $\boldsymbol{\Delta H}_{\text {elec }}^{\mathbf{0 . 5} \boldsymbol{c}}$ & $\boldsymbol{\Delta H}_{\text {elec }}^{\mathbf{0 . 7 5 , c}}$ & $\boldsymbol{\Delta H}_{\text {elec }}^{\text {ref }}$ & Benchmarked IPEA \\
\hline $\mathbf{1}$ & -39.0 & -16.8 & 1.7 & 17.7 & 6.9 & 0.57 \\
$\mathbf{2}$ & -46.1 & -22.5 & -3.7 & 12.9 & 7.6 & 0.66 \\
$\mathbf{3}$ & -41.7 & -18.0 & 0.7 & 17.1 & 5.9 & 0.57 \\
$\mathbf{4}$ & -24.2 & -0.8 & 18.0 & 34.5 & 23.2 & 0.57 \\
$\mathbf{5}$ & -7.8 & 15.0 & 33.4 & 49.8 & 22.9 & 0.35 \\
$\mathbf{6}$ & -23.3 & -0.1 & 18.7 & 35.3 & 25.9 & 0.60 \\
$\mathbf{7}$ & -14.8 & 6.9 & 24.5 & 40.2 & 15.9 & 0.37 \\
\hline Average & \multicolumn{7}{|c}{}
\end{tabular}

Table 3: Comparison between the reference adiabatic energy values $\left(\Delta H_{\text {elec }}^{\text {ref }}\right)$ and those obtained with IPEA $=0.53$ a.u. and the corrected (c) basis set. Agreement in the description of the ground state is displayed with $\boldsymbol{V}$ or $\boldsymbol{X}$ (in brackets). All energy values are given in $\mathrm{kJ} \cdot \mathrm{mol}^{-1}$.

\begin{tabular}{cccc}
\hline & $\boldsymbol{\Delta H}_{\text {elec }}^{\mathbf{0 . 5 3} \boldsymbol{c}}$ & $\boldsymbol{\Delta H}_{\text {elec }}^{\text {ref }}$ (Spin State) & Error \\
\hline $\mathbf{1}$ & 3.9 & $6.9(\checkmark)$ & -3.0 \\
$\mathbf{2}$ & -1.3 & $7.6(\times)$ & -8.9 \\
$\mathbf{3}$ & 3.1 & $5.9(\checkmark)$ & -2.8 \\
$\mathbf{4}$ & 20.4 & $23.2(\checkmark)$ & -2.8 \\
$\mathbf{5}$ & 35.8 & $22.9(\checkmark)$ & +12.9 \\
$\mathbf{6}$ & 21.1 & $25.9(\checkmark)$ & -4.8 \\
$\mathbf{7}$ & 26.8 & $15.9(\checkmark)$ & +10.9 \\
\hline Mean Absolute Error & & & $\mathbf{6 . 6}$
\end{tabular}




\section{References and Notes}

phen $=1,10$-phenanthroline,

abpt= 4-amino-3,5-bis(pyridin-2-yl)-1,2,4- triazole

babpby $=\mathrm{N}-(6-(6-($ pyridin-2-ylamino)pyridin-2-yl)pyridin-2-yl)pyridin-2-amine

$\mathrm{pz}=$ pyrazine

bipy= 2,2'-bipyridine

1-bpp=2,6-di(pyrazol-1-yl)pyridine

\section{Acknowledgements}

Contract grant sponsor: Spanish Government (to M.F. and J.R.-A.); Contract grant number: MAT201125972 and MAT2014-54025-P; Contract grant sponsor: "Ramón y Cajal" Fellowship (to J.R.-A.); Contract grant sponsor: University of Barcelona (to M.F.); Contract grant sponsor: BSC, CSUC, and Catalan DURSI; Contract grant number: 2014SGR1422; Contract grant sponsor: LabEx-Chemistry of Complex Systems (to S.V.); Contract grant number: ANR-10-LABX-0026_CSC 


\section{References}

[1] M. A. Halcrow, Spin-Crossover Materials: Properties and Applications; John Wiley \& Sons Ltd, Oxford, UK 2013.

[2] P. Gütlich, H. A. Goodwin, Spin Crossover in Transition Metal Compounds I; Springer, Berlin/Heidelberg 2004.

[3] A. Bousseksou, G. Molnar, L. Salmon, W. Nicolazzi, Chem. Soc. Rev. 2011, 40, 3313.

[4] G. A. Craig, O. Roubeau, G. Aromí, Coord. Chem. Rev. 2014, 269, 13.

[5] P. Gütlich, A. Hauser, H. Spiering, Angew. Chem. Int. Ed. Engl. 1994, 33, 2024.

[6] P. Gutlich, Y. Garcia, H. A. Goodwin, Chem. Soc. Rev. 2000, 29, 419.

[7] A. Rudavskyi, C. Sousa, C. de Graaf, R. W. A. Havenith, R. Broer, J. Chem. Phys. $2014,140,184318$.

[8] A. Rudavskyi, R. W. A. Havenith, R. Broer, C. de Graaf, C. Sousa, Dalton Trans. $2013,42,14702$.

[9] B. Ordejon, C. de Graaf, C. Sousa, J. Am. Chem. Soc. 2008, 130, 13961.

[10] G. Ghigo, B. O. Roos, P. Malmqvist, Chem. Phys. Lett. 2004, 396, 142.

[11] M. Kepenekian, V. Robert, B. Le Guennic, J. Chem. Phys. 2009, 131, 114702.

[12] L. M. Lawson Daku, F. Aquilante, T. W. Robinson, A. Hauser, J. Chem. Theory Comput. 2012, 8, 4216.

[13] S. Vela, M. Fumanal, J. Ribas-Arino, V. Robert, Phys. Chem. Chem. Phys. 2015, $17,16306$.

[14] For more information, see: http://www.molcas.org/ANO/.

[15] G. Karlström, R. Lindh, P. Malmqvist, B. O. Roos, U. Ryde, V. Veryazov, P. O. Widmark, M. Cossi, B. Schimmelpfennig, P. Neogrady, L. Seijo, Comput. Mater. Sci. 2003, 28, 222.

[16] B. Gallois, J. A. Real, C. Hauw, J. Zarembowitch, Inorg. Chem. 1990, 29, 1152.

[17] J. A. Real, A. B. Gaspar, M. C. Munoz, Dalton Trans. 2005, 2062.

[18] S. Bonnet, M. A. Siegler, J. S. Costa, G. Molnar, A. Bousseksou, A. L. Spek, P. Gamez, J. Reedijk, Chem. Commun. (Cambridge, UK) 2008, 5619. 
[19] L. Salmon, G. Molnar, S. Cobo, P. Oulie, M. Etienne, T. Mahfoud, P. Demont, A. Eguchi, H. Watanabe, K. Tanaka, A. Bousseksou, New J. Chem. 2009, 33, 1283.

[20] J. A. Real, M. C. Muñoz, J. Faus, X. Solans, Inorg. Chem. 1997, 36, 3008.

[21] J. M. Holland, J. A. McAllister, Z. Lu, C. A. Kilner, M. Thornton-Pett, M. A. Halcrow, Chem. Commun. (Cambridge, UK) 2001, 577.

[22] P. Giannozzi, S. Baroni, N. Bonini, M. Calandra, R. Car, C. Cavazzoni, D. Ceresoli, G. L. Chiarotti, M. Cococcioni, I. Dabo, A. D. Corso, S. Gironcoli, S. Fabris, G. Fratesi, R. Gebauer, U. Gerstmann, C. Gougoussis, A. Kokalj, M. Lazzeri, L. Martin-Samos, N. Marzari, F. Mauri, R. Mazzarello, S. Paolini, A. Pasquarello, L. Paulatto, C. Sbraccia, S. Scandolo, G. Sclauzero, A. P. Seitsonen, A. Smogunov, P. Umari, R. M. Wentzcovitch, J. Phys. Condens. Matter 2009, 21, 395502.

[23] M. Kepenekian, V. Robert, B. Le Guennic, C. De Graaf, J. Comput. Chem. 2009, $30,2327$.

[24] K. Pierloot, S. Vancoillie, J. Chem. Phys. 2006, 125, 124303.

[25] K. Andersson, P. A. Malmqvist, B. O. Roos, A. J. Sadlej, K. Wolinski, J. Phys. Chem. $1990,94,5483$.

[26] B. O. Roos, R. Lindh, P. Malmqvist, V. Veryazov, P. O. Widmark, J. Phys. Chem. A $2005,109,6575$.

[27] P. O. Widmark, P. Malmqvist, B. Roos, Theory Chim. Acta 1990, 77, 291.

[28] B. O. Roos, R. Lindh, P. Malmqvist, V. Veryazov, P. O. Widmark, J. Phys. Chem. A 2003, 108, 2851.

[29] S. Vela, J. J. Novoa, J. Ribas-Arino, Phys. Chem. Chem. Phys. 2014, 16, 27012.

[30] N. Queralt, D. Taratiel, C. de Graaf, R. Caballol, R. Cimiraglia, C. Angeli, J. Comput. Chem. 2008, 29, 994.

[31] C. Angeli, R. Cimiraglia, J. P. Malrieu, J. Chem. Phys. 2002, 117, 9138.

[32] C. Angeli, R. Cimiraglia, J. P. Malrieu, Chem. Phys. Lett. 2001, 350, 297.

[33] C. Angeli, R. Cimiraglia, S. Evangelisti, T. Leininger, J. P. Malrieu, J. Chem. Phys. 2001, 114, 10252. 